\title{
Design of IIR Filters with Definite Group Delay (Lowpass Equalization) using LabVIEW
}

\author{
Abhay kumar singh ${ }^{1}$ and Lalit singh garia ${ }^{2}$ \\ ${ }^{1}$ M.tech scholar Department of Electronics and Communication Engineering \\ B.T.K.I.T Dwarahat Almora 263653, Uttarakhand, India \\ ${ }^{2}$ Assistant Professor Department of Electronics and Communication Engineering \\ B.T.K.I.T Dwarahat Almora 263653, Uttarakhand, India \\ ${ }^{1}$ Abhays308@gmail.com, ${ }^{2}$ lalit.s.garia@gmail.com
}

\begin{abstract}
Digital filters with lined phase responses that is constant group delay responses are desirable in many uses for signal treating. In this paper, a new method is proposed for scheming maximally smooth IIR filters with constant group delay in the pass band. This paper presents a concept of IIR filters with equalized group delay response. The group delay has been equalized by all pass filter. In this IIR filter design some constant group delay has been introduce and effect of group delay on phase is studied. At some extent of frequency group delay of compensated filter is linear so that phase of the IIR filter is linear at particular range of frequency. This paper looks at a compromise in which the IIR filter is followed by a allpass filter, which adaptively tries to linearize the overall phase response but leaves the magnitude response unchanged.
\end{abstract}

Keywords: group delay, allpass filter, IIR filter, compensated filter

\section{Introduction}

Digital signal dispensation takes developed the practical apparel of electronics. Signals produce in the normal method in the actual world. Therefore signals essential to be treated so that the data they cover can be showed, analyzed or changed to alternative type of signals. In the real world analog signals such as sound, light, temperature or pressure and manipulate them [2]. Earlier digital signal process the signal next from the real world needs to be converted in to the digital form. Therefore convert these actual signals into the setup of Zeros and Ones. Infinite impulse response (IIR) filters that you design using Butterworth, Chebyshev, or Elliptic methods usually have a nonconstant group delay, which means that they require nonlinear phase or phase distortion. The greatest deviation from a continuous group delay classically occurs at the verge of the passband or someplace in the change band. Given a filter with phase alteration we can cascade the sieve with an allpass filter to linear the phase retort in the stated frequency ranges though keeping the magnitude response unaffected.

\section{IIR Filter}

The butterworth channel has a greatly level reaction, i.e. no passband swell and move off of short $20 \mathrm{db}$ for every shaft. Extra name for it is level to a great degree size channels at the recurrence of $\Omega=0$, as the initial $2 \mathrm{~N}-1$ subordinates of the exchange capacity when $\Omega=0$ are equivalent to zero. The Butterworth channels accomplish its evenness to the detriment of a moderately broad move district from passband to stopband with normal transient qualities. This channel is totally characterized scientifically by two variables that is cut off recurrence and number of posts. Contrasted with chebyshev channel, the stage linearity of butterworth channel is better [1]. As such, the gathering delay (subordinate of 
stage with reverence to recurrence) is more consistent regarding recurrence. This implies the waveform mutilation of the butterworth channel is lesser. This Butterworth channels have the accompanying qualities.

\section{Phase Response Non-Linearity}

The outline issue of the IIR channels is normally communicated as the guess issue of the greatness and the stage reactions. Notwithstanding, the gathering delay reaction of the channel got by those techniques has a tendency to wind up moderately substantial, especially in the territory of the band-edge. This is on the grounds that that the perplexing extent mistake, which incorporate both the size and stage reactions, is diminished rather than the gathering delay blunder. By and large, an all pass channel is utilized to adjust the stage reaction to make the gathering postpone around steady in the intrigued transmission capacity. Be that as it may, the utilization of the all pass channel is not basically a decent procedure on the grounds that the channel coefficients are repetitive. In this way, it is expected to understand the channel that has an evened out gathering delay without utilizing the all pass channel [3-4].

This moves the examination on planning the IIR channels with determined most extreme gathering delay mistakes by specifically approximating the gathering delay reaction. Computerized channel outline, as with all other designing practices, includes tradeoffs. For instance Finite Impulse Response (FIR) channels are straightforward, and can be intended to give a direct stage reaction or steady gathering delay. Interminable Impulse Response (IIR) channels may accomplish the same levels of constriction as FIR channels with lesser coefficients. group delay change is a sensible differentiating choice to compensate non-direct stage response affects, an issue of growing eagerness, for case, in cutting edge quick signal taking care of, where different tight stations are suited in the accessible recurrence band. IIR elliptic channels have the downside of having exceedingly non-direct stage reaction, notwithstanding their leeway as far as computational intricacy, when contrasted with straight stage FIR filters [3-4]. Then again, as roughly straight stage trademark may fulfill framework necessities in numerous applications, stage leveled IIR elliptic channels may discover an edge over contending direct stage FIR partners The Butterworth topology tries to smooth the recurrence reaction in the passband however much as could reasonably be expected while diminishing the move band and stop band monotonically [6-9]. Group delay has been define as

$$
\tau(w)=\operatorname{grd}\left[H\left(e^{j \omega}\right)\right]=-\frac{d}{d w}\left(\arg \left[H\left(e^{j \omega}\right)\right]\right)=\alpha
$$

And linear phase of general form

$$
\arg \left[H\left(e^{j \omega}\right)\right]=\beta-\omega \alpha \quad 0 \leq \omega<\pi
$$

Simple all-pass system is define by the equations

$$
\begin{gathered}
H_{a p}(z)=\frac{z^{-1}-a^{*}}{1-a z^{-1}} \\
H_{a p}\left(e^{j \omega}\right)=\frac{e^{-j \omega}-a^{*}}{1-a e^{-j \omega}}=e^{-j \omega} \frac{1-a^{*} e^{j \omega}}{1-a e^{-j \omega}}
\end{gathered}
$$

Consequently that most overall procedure with actual instinct response

$$
H_{a p}(z)=A \prod_{k=1}^{M_{r}} \frac{z^{-1}-d_{k}}{1-d_{k} z^{-1}} \prod_{k=1}^{M_{c}} \frac{\left(z^{-1}-c_{k}^{*}\right)\left(z^{-1}-c_{k}\right)}{\left(1-c_{k} z^{-1}\right)\left(1-c_{k}^{*} z^{-1}\right)}
$$


Where A: positive constant, $\mathrm{d}_{\mathrm{k}}$ : real poles, $\mathrm{c}_{\mathrm{k}}$ : complex poles The group delay of this system can be written as

$$
\operatorname{grd}\left[\frac{e^{-j \omega}-r e^{-j \theta}}{1-r e^{j \theta} e^{-j \omega}}\right]=\frac{1-r^{2}}{1-2 r \cos (\omega-\theta)+r^{2}}=\frac{1-r^{2}}{\left|1-r e^{j \theta} e^{-j \omega}\right|^{2}}
$$

We know that For steady and causal method $|\mathrm{r}|<1$ and Group delay of all-pass systems is continuously affirmative.

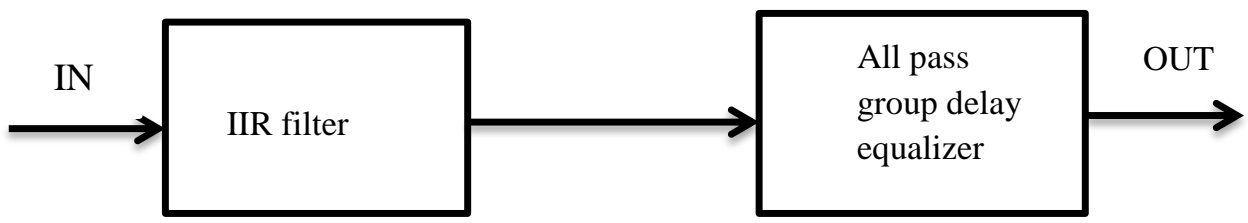

Figure 1. Group Delay Equalization of IIR Filters

\section{Results And Discussion}

Specifications taken for the design of Butterworth,chebyshev and Elliptic filter are:

Sampling frequency $=10000 \mathrm{~Hz}$.

Passband frequency $=500 \mathrm{~Hz}$

Stopband frequency $=1000 \mathrm{~Hz}$

Passband ripples $A_{p}=15 \mathrm{db}$

Stopband ripples $A_{s}=100 \mathrm{db}$

The coefficient of butterworth filter is calculated by the specification of the butterworth filter topology. The TF of the Butterworth filter is

$$
\mathrm{TF}=\frac{0.1367 \mathrm{z}^{-1}+0.1367 \mathrm{z}^{-2}+0.0223 \mathrm{z}^{-4}+0.447 \mathrm{z}^{-5}+0.223 \mathrm{z}^{-6}+0.0196 \mathrm{z}^{-7}}{0.72650 \mathrm{z}^{-1}-1.736 \mathrm{z}^{-3}+0.8257 \mathrm{z}^{-4}-1.5217 \mathrm{z}^{-5}+0.600 \mathrm{z}^{-6}}
$$

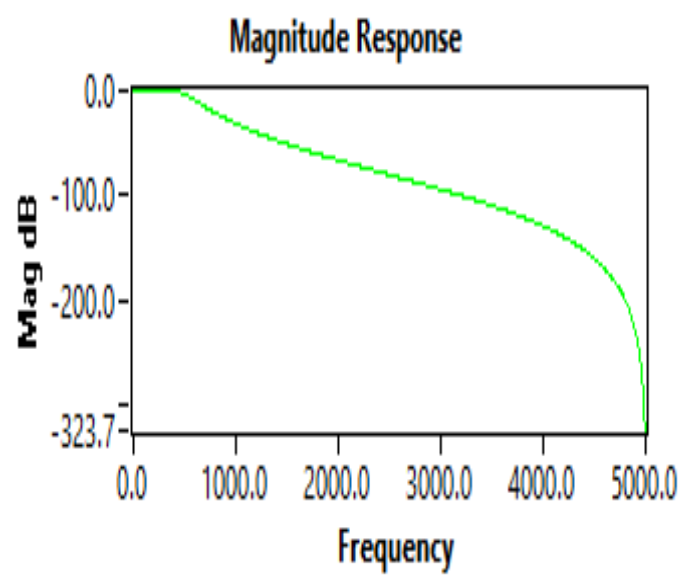




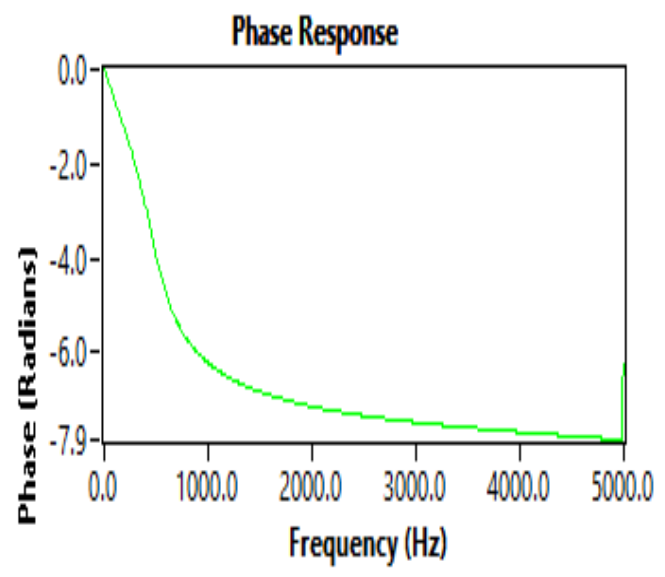

Figure 2. The Magnitude and Phase Response of the Butterworth filter of Order 5 using Labview

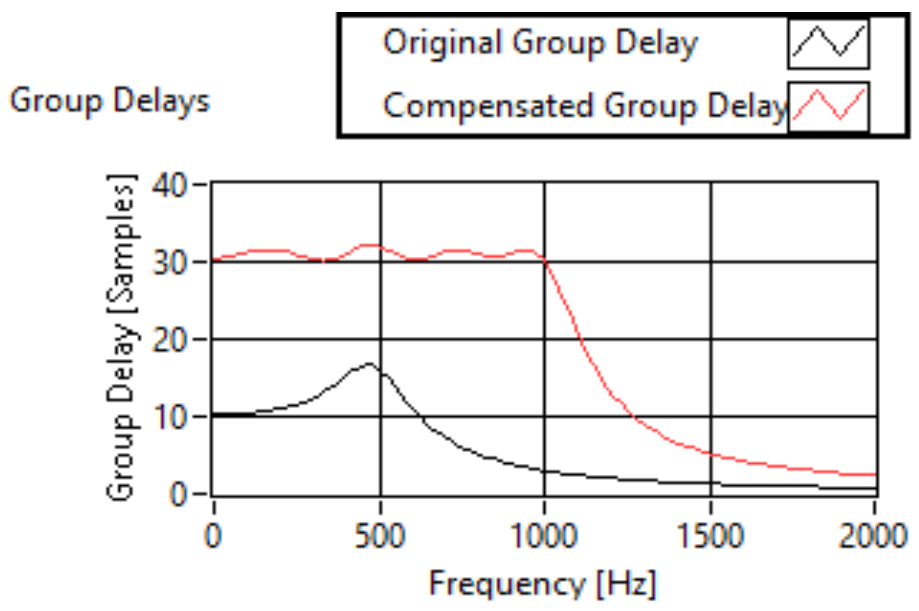

Figure 3. The Group Delay of Filter and the Equalize Group Delay of Butterworth Filter Using Labview

The coefficient of Chebyshev filter is calculated by the specification of the Chebyshev filter topology. The TF of the Chebyshev filter is

$$
\mathrm{TF}=\frac{0.0094 \mathrm{z}^{-1}+0.0094 \mathrm{z}^{-2}+0.0183 \mathrm{z}^{-4}+0.0368 \mathrm{z}^{-5}+0.0183 \mathrm{z}^{-6}}{-0.9812 \mathrm{z}^{-1}-1.9080 \mathrm{z}^{-3}+0.9815 \mathrm{z}^{-4}}
$$



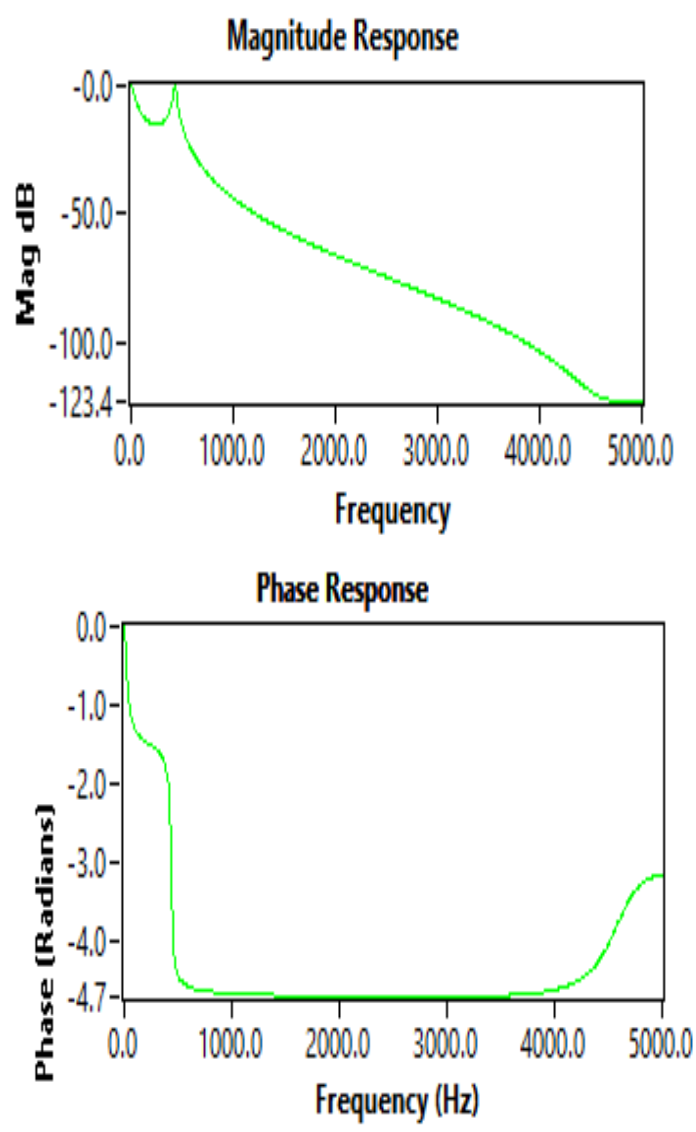

Figure 4. The Magnitude and Phase Response of the Chebyshev Filter using Labview

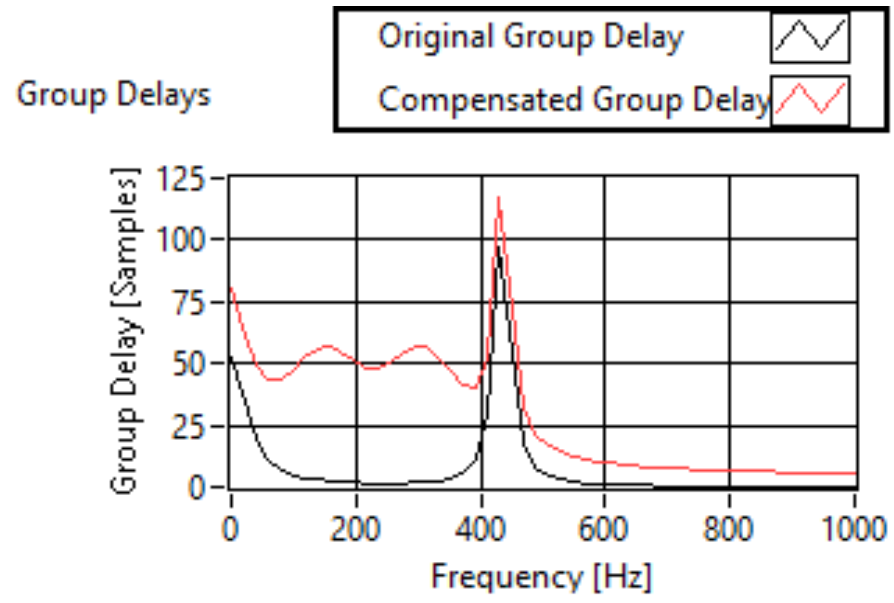

Figure 5. The Group Delay of Filter and the Equalize Group Delay of Chebyshev Filter Using Labview

Specifications taken for the design of Inverse Chebyshev filter are:

Sampling frequency $=1000 \mathrm{~Hz}$.

Passband frequency $=100 \mathrm{~Hz}$

Stopband frequency $=300 \mathrm{~Hz}$

Passband ripples $A p=10 \mathrm{db}$

Stopband ripples $\mathrm{As}=40 \mathrm{db}$ 
The coefficient of inverse Chebyshev filter is calculated by the specification of the Inverse Chebyshev filter topology.The TF of the inverse Chebyshev filter is

$$
\mathrm{TF}=\frac{0.1027 \mathrm{z}^{-1}+0.1027 \mathrm{z}^{-2}++0.0869 \mathrm{z}^{-4}-0.1310 \mathrm{z}^{-5}+0.869 \mathrm{z}^{-6}}{0.794 \mathrm{z}^{-1}-1.769 \mathrm{z}^{-3}+0.8124 \mathrm{z}^{-4}}
$$
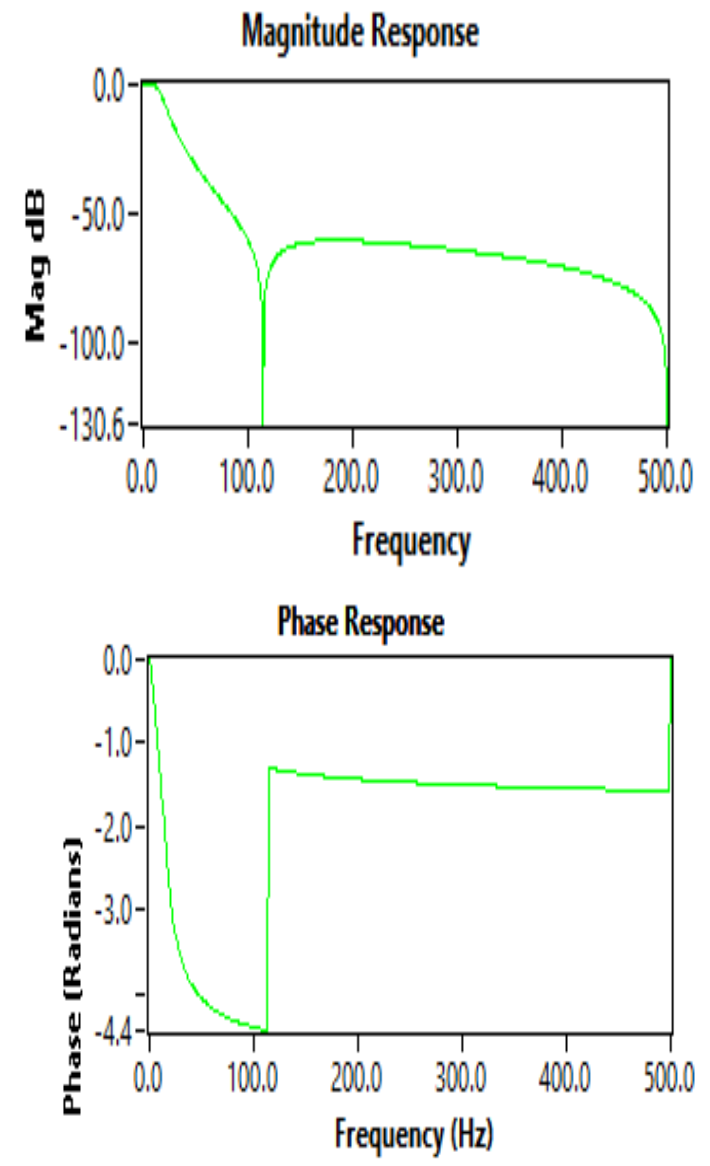

Figure 6. The Magnitude and Phase Response of the Inverse Chebyshev Filter Using Labview

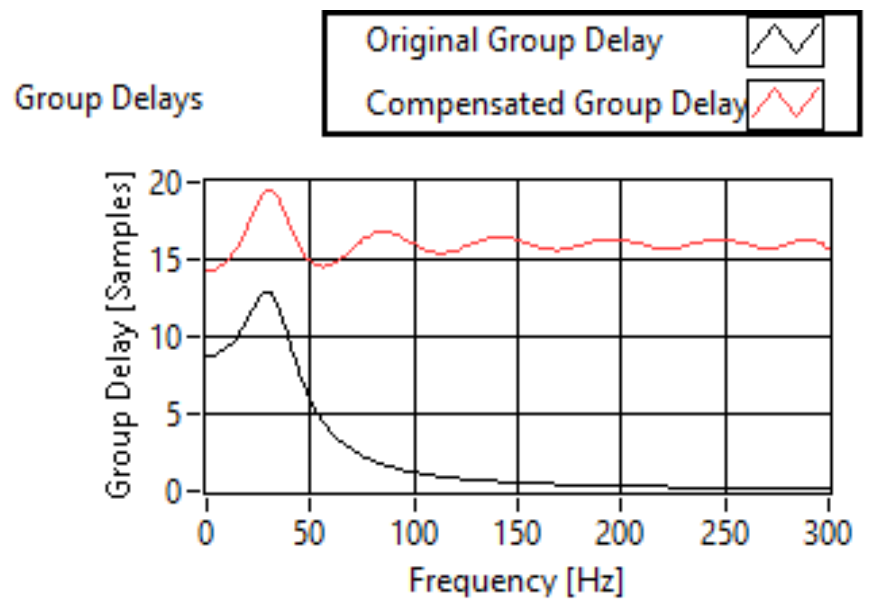

Figure 7. The Group Delay of Filter and the Equalize Group Delay of Inverse Chebyshev Filter Using Labview 
The coefficient of Elliptic Filter is calculated by the specification of the Elliptic Filter topology. The transfer function of the Elliptic Filter is

$$
\mathrm{TF}=\frac{0.0094 \mathrm{z}^{-1}+.0094 \mathrm{z}^{-2}-0.0235 \mathrm{z}^{-4}+.0267 \mathrm{z}^{-5}+.0235 \mathrm{z}^{-6}}{-0.9811 \mathrm{z}^{-1}-1.9080 \mathrm{z}^{-2}+0.9816 \mathrm{z}^{-4}}
$$
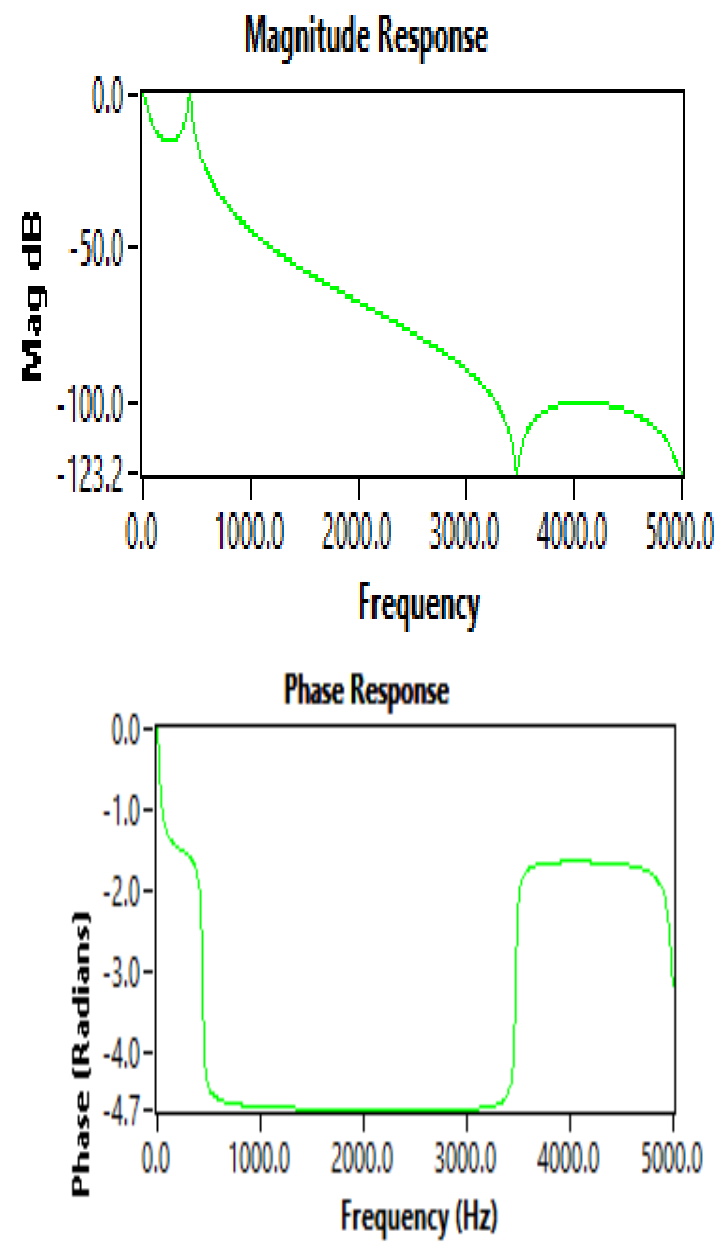

Figure 8. The Magnitude and Phase Response of the Elliptic Filter using Labview

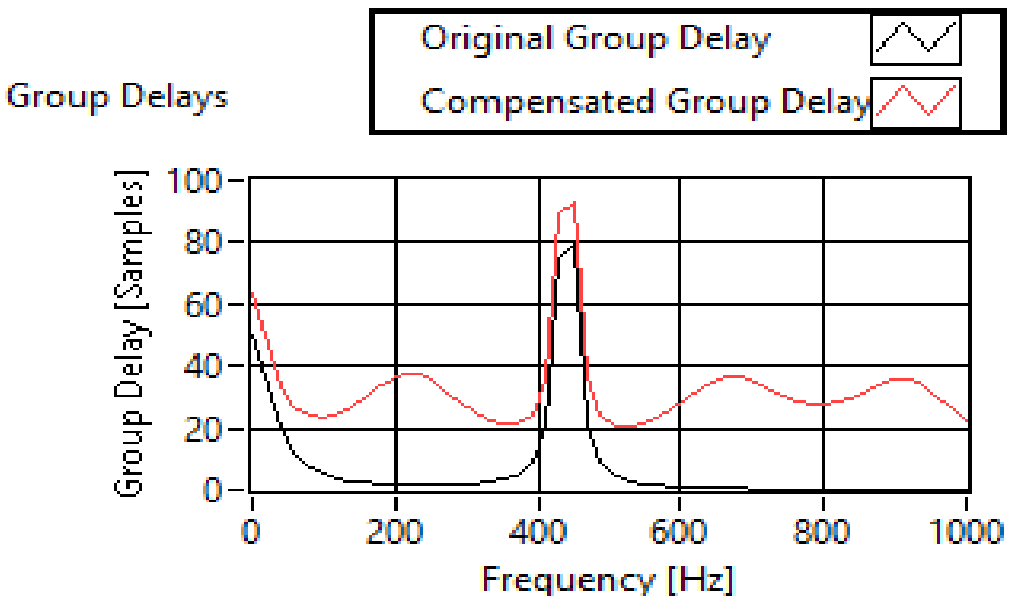

Figure 9. Show the Group Delay of Filter and the Equalize Group Delay of Elliptic Filter Using Labview 


\section{Conclusion}

The phase response achieved an approximately constant group delay in the pass band. The ripples were found to decrease as the equalizer order was made greater and the delay line length was also made larger. The ripples were observed to have a extreme at the pass band edge and were a least when the equalizer order was the same as the length of the delay line. They were not totally eliminated even as the order was made large.In LabVIEW the factors can be alteration at the period of accomplishment of the program but in case of MATLAB it is not imaginable. LabVIEW Design is originated on Graphical Software design so that the analysis of the presentation can be done very easily. In LabVIEW examination of all kinds of Filters (Lowpass, HighPsss, Bandpass, and Bandstop) is possible in single program. It is additionally confirmed that on expanding the request of any filter the move band diminishes for the similar limitations. The steady estimation of the group delay shows that the repaid channel straightly approaches the stage reaction in the passband. However,compared to the first filter, the repaid channel likewise builds the postponement and separating calculation.

\section{References}

[1] A. Jiang and H. K. Kwan, "IIR Digital Filter Design with Novel Stability Criterion Based on Argument Principle", Department of Electrical and Computer Engineering,University of Windsor, vol. 1, (2007), pp. 126-131.

[2] J. Y. Beyon, "Hands-On Exercise Manual for LabVIEW Programming, Data Acquisition and Analysis", Prentice Hall, Inc., New Jersey, (2001).

[3] M. L. Chugani, "LabVIEW Signal Processing", Prentice Hall, Inc., Upper Saddle River, New Jersey, (1998).

[4] C. L. Clark, "LabVIEW Digital Signal Processing and Digital Communications", Tata McGRAWHILL, (2005).

[5] M. F. Fahmy, M. A. Zahhad and M. I. Shoby, "Design of Selective Linear Phase Switched-Capacitor Filters with Equiripple Passband Amplitude Responses", IEEE Trans. On Circuits and Systems, CAS35, no. 10, (1988), pp. 1220-1229.

[6] L. B. Jackson, "Digital Filters and Signal Processing", $3^{\text {rd }}$ ed., Kluwer Academic Publishers, (1996).

[7] N. Kim, "Digital Signal Processing System-Level Design Using LabVIEW", Elsevier Inc., vol. 1, (2005), pp. 122-127.

[8] A. V. Oppenheim and R. W. Schafer, "Discrete Time Signal Processing", 2nd ed., Pearson Education, (2005).

[9] J. G. Proakis and D. G. Manolakis, "Digital Signal Processing, Principles Algorithms, and Applications", $3^{\text {rd }}$ ed., Pearson Education, Inc., (1996).

[10] L. K. Wells and J. Travis, "LabVIEW for Everyone Graphical Programming Made Even Easier", Prentice Hall, Inc., Upper Saddle River, New Jersey, (1997). 